Pacific

Journal of

Mathematics

A CANCELLATION FORMULA OF ALVAREZ-GAUMÉ AND WITTEN AND A SIGNATURE DEFECT-TYPE INVARIANT OF 11-DIMENSIONAL LENS SPACES WITH SPIN STRUCTURES

YOSHIHIRO FUKUMOTO

Volume $235 \quad$ No. 2

April 2008 


\title{
A CANCELLATION FORMULA OF ALVAREZ-GAUMÉ AND WITTEN AND A SIGNATURE DEFECT-TYPE INVARIANT OF 11-DIMENSIONAL LENS SPACES WITH SPIN STRUCTURES
}

\author{
YOSHIHIRO FUKUMOTO
}

\begin{abstract}
In this paper, we define an integer-valued diffeomorphism invariant of 11dimensional lens spaces with spin structures. This invariant modulo 16 gives the generalized Rohlin invariant and is defined in a way analogous to the signature defects by using a cancellation formula which discovered by L. Alvarez-Gaumé and E. Witten in their study of gravitational anomalies. In particular, we give an explicit formula for the invariant by using the Kawasaki $V$-index theorem, and we calculate the invariant for several examples of lens spaces. Using this formula, we obtain a necessary condition for smooth 11-dimensional free $\mathbb{Z} / p$-spheres to be the boundaries of 12dimensional free spin $\mathbb{Z} / p$-manifolds. We also prove that this invariant has a reciprocity property similar to the reciprocity law of the Theta multiplier given by $B$. Berndt.
\end{abstract}

\section{Introduction}

In this paper, we will define an integer-valued diffeomorphism invariant of 11dimensional lens spaces with spin structures. This invariant modulo 16 gives the generalized Rohlin invariant and is defined in a way analogous to the signature defects by using a cancellation formula of L. Alvarez-Gaumé and E. Witten [1984] concerning the gravitational anomalies. In particular, we calculate an explicit formula for this invariant by using the Atiyah-Singer-Kawasaki $V$-index formula, and we prove a reciprocity formula of this invariant.

The cancellation formula relates $\hat{A}$-genera and $L$-genera in dimension 12 , and hence the signature-defect type invariant gives an invariant in dimension 11 . Using the determination of the structure of the spin cobordism ring by D. W. Anderson, E. H. Brown, and F. P. Peterson [Anderson et al. 1967], the spin cobordism ring in dimension 11 is calculated to be trivial. Hence the invariant is integer-valued and in fact gives an integral lift of the generalized Rohlin invariant of 11-dimensional lens

MSC2000: primary 58J28, 58J20, 11A15; secondary 55N22, 53C27.

Keywords: index theorem, $V$-manifold, reciprocity law, generalized Rohlin invariant, lens space.

Research supported by MEXT Grant-in-Aid for Scientific Research (18740039). 
spaces with spin structures. In particular, we use the Kawasaki $V$-index theorem to exhibit as a trigonometric sum the invariant for 11-dimensional lens spaces with spin structures. Using this formula, we obtain a necessary condition for smooth 11-dimensional free $\mathbb{Z} / p$-spheres to be the boundaries of 12-dimensional free spin $\mathbb{Z} / p$-manifolds. We also prove that the invariant has a reciprocity property similar to that of an arithmetic sum appearing in the transformation law, given by B. Berndt [1978], of the classical theta function. In fact, this reciprocity property follows from an easy calculation using the residue theorem.

Reciprocity properties of trigonometric sums have their origin in the reciprocity law of the Dedekind sum, which follows from the transformation properties of the famous Dedekind $\eta$-function. F. Hirzebruch and D. Zagier [1974] gave a geometric interpretation of the Dedekind sums using the Atiyah-Singer index theorem for the signature operators, and Zagier [1972] extended these arguments to give a reciprocity law for his higher-dimensional Dedekind sums.

In Seiberg-Witten gauge theory, M. Furuta [2001] developed a technique of finite-dimensional approximation for the Seiberg-Witten monopole equation in order to prove the 10/8-inequality for closed spin 4-manifolds. On the other hand, by using a relation of the characteristic classes, namely, that in 4 dimensions the $L$-genus is equal to -8 times the $\hat{A}$-genus, we can define a signature defect-type invariant of 3-dimensional manifolds, which we call the $w$-invariant [Fukumoto and Furuta 2000]. This invariant is an integral lift of the Rohlin invariant and can in fact be shown to be equal to the Neumann-Siebenmann invariant for plumbed homology 3 -spheres by taking the auxiliary $4-V$-manifold to be the associated plumbed 4- $V$-manifold. Using a $V$-manifold version of the $10 / 8$-inequality, we can prove that this invariant has a homology spin cobordism invariance in a certain class of homology 3-spheres, including a class of Seifert homology 3-spheres. In particular, the singular point contribution of the $w$-invariant satisfies a reciprocity property [Fukumoto et al. 2001; Fukumoto 2002] which is essentially identical to that of the arithmetic sum appearing in the transformation law, given by [Berndt 1978], of the logarithm of the classical theta functions.

Furthermore, M. Ue [2001] defined an invariant of spherical 3-manifolds, called the $\delta$-invariant, to be the singular point contribution of the $w$-invariants; Ue accomplished this by using the cone over spherical 3-manifolds. Ue also derived certain constraints on the intersection forms of spin 4-manifolds bounded by spherical 3 -manifolds. Our invariant can be seen as an 11-dimensional version of the $\delta$ invariant.

K. Liu [1995] and F. Han and W. Zhang [2004] generalized the miraculous cancellation formula of Alvarez-Gaumé and Witten to another one in dimension $8 k+4$. We can perform a similar construction of invariants for $(8 k+3)$-dimensional spin manifolds. In fact, K. Liu derived an expression of the generalized Rohlin 
invariant for $(8 k+3)$-dimensional closed spin manifolds by using the $\eta$-invariant to prove that this generalized Rohlin invariant is a spectral invariant.

Our approach to defining the invariant is different: instead of using the $\eta$ invariant, which depends on the metric, we use $V$-indices, which depend on the choice of auxiliary bounding $V$-manifolds. In future work, we will investigate reciprocity properties of the invariant for $(8 k+3)$-dimensional lens spaces.

This paper is organized as follows. In Section 2 we define a diffeomorphism invariant of 11-dimensional lens spaces with spin structures by using the formula of Alvarez-Gaumé and Witten; we prove that this invariant gives the generalized Rohlin invariant modulo integers 16. Subsequently, we employ the generalized Rohlin invariants for higher dimensional spin manifolds in order to show that our invariant gives an integral lift of the Rohlin invariant of 11-dimensional lens spaces. To calculate the invariant explicitly, we describe the set of all $\operatorname{spin}^{c}$ structures on lens spaces in Section 3. In Section 4, we use the Kawasaki $V$-index formula [1981] to give an explicit formula for the invariant of 11-dimensional lens spaces with spin structures. We give several examples in Section 5. Finally, in Section 6 we prove a reciprocity property of the invariant of 11-dimensional lens spaces with spin structures by using the residue formula; this gives a $V$-index formula on the 12-dimensional weighted projective spaces. Appendix A evaluates several characteristic numbers of weighted projective spaces, in the sense of $V$-manifolds, by using the formalism of toric varieties.

\section{A signature defect-type invariant of 11-dimensional lens spaces with spin structures}

Here, we define an integer-valued diffeomorphism invariant of 11-dimensional lens spaces with spin structures. The definition is analogous to the invariant of signature defects, and uses the following formula, which was discovered by L. AlvarezGaumé and E. Witten [1984] while studying of gravitational anomalies.

Theorem 1. Let $M$ be a 12-dimensional manifold. Let $L(M), \hat{A}(M)$, and $\hat{A}(M, T)$ be the L-genus, $\hat{A}$-genus, and $\hat{A}$-genus twisted by the tangent bundle of the manifold $M$, respectively. Then $L(M)=8 \hat{A}(M, T)-32 \hat{A}(M)$.

By using this equality, we define an invariant $\delta_{A W}$ for the pair $\left(Y, c_{Y}\right)$ of 11dimensional lens space $Y$ and its spin structure $c_{Y}$. Then (2n-1)-dimensional lens space is a $(2 n-1)$-dimensional smooth manifold $L(p ; \vec{a})$ determined as follows. Choose an integer $p$ and an $n$-tuple of integers $\vec{a}=\left(a_{1}, \ldots, a_{n}\right)$ coprime to $p$. Then $L(p ; \vec{a})$ is defined as the quotient of the $(2 n-1)$-dimensional unit sphere $S^{2 n-1} \subset \mathbb{C}^{n}$ by the $\mathbb{Z} / p$ action on $\mathbb{C}^{n}$ given by $\zeta \cdot\left(z_{j}\right)=\left(\zeta^{a_{j}} z_{j}\right)$ with $\zeta^{p}=1$. On the other hand, let $X(p ; \vec{a})$ be the quotient of the $2 n$-dimensional unit ball $B^{2 n} \subset \mathbb{C}^{n}$ by the $\mathbb{Z} / p$ action. Then $X(p ; \vec{a})$ is a $2 n$-dimensional $V$-manifold with 
an isolated singularity at the origin $[(0, \ldots, 0)]$; its boundary is diffeomorphic to the lens space $L(p ; \vec{a})$. For definitions and several facts about $V$-manifolds, see [Satake 1957].

By the result on structure of the spin cobordism ring by D. W. Anderson, E. H. Brown and F. P. Peterson [Anderson et al. 1967], we know

Lemma 2. The spin cobordism group in dimension 11 is trivial, that is, $\Omega_{11}^{\text {spin }}=0$. Proof. The spin cobordism ring $\Omega_{*}^{\text {spin }}$ is the homotopy group $\pi_{*}(M$ spin $)$ of the spectrum ( $M$ spin), and the homotopy group is the product of the spectra of the bo part and the $K(\mathbb{Z} / 2, \cdot)$ part. The bo part can be calculated by using Bott periodicity and by using that its $\pi_{m}$ is nontrivial for $m \geq 0$ with $m \equiv 0,1,2,4(\bmod 8)$. The $K(\mathbb{Z} / 2, d)$ part can be calculated indirectly: We use the Poincaré polynomial of $H^{*}(M$ spin $)$ and its decomposition into $b o$ and $K(\mathbb{Z} / 2, \cdot)$ parts to see that the Poincaré polynomial of the $K(\mathbb{Z} / 2, \cdot)$ part vanishes up to degree 20.

Let $\left(Y, c_{Y}\right)$ be a pairing of a spin 11-dimensional lens space $Y=L(p ; \vec{a})$ and its spin structure $c_{Y}$. Since $\Omega_{11}^{\text {spin }}$ is trivial, that is, $\left[\left(Y, c_{Y}\right)\right]=0 \in \Omega_{11}^{\text {spin }}$, there exists a 12-dimensional spin manifold $\left(W, c_{W}\right)$ with boundary $\partial\left(W, c_{W}\right)=-\left(Y, c_{Y}\right)$. Then by taking the auxiliary $V$-manifold $X=X(p ; \vec{a})$ associated to the lens space $Y=L(p ; \vec{a})$, we define an integer-valued invariant of the lens space $\left(Y, c_{Y}\right)$ as follows.

\section{Definition 3.}

$$
\delta_{A W}\left(Y, c_{Y}\right):=8 \operatorname{index}_{V}(\mathscr{D} \otimes T)\left(X \cup_{Y} W\right)-32 \operatorname{index}_{V} \mathscr{D}\left(X \cup_{Y} W\right)-\operatorname{Sign}(W),
$$

where $T=T\left(X \cup_{Y} W\right) \otimes \mathbb{C}$.

The following shows that $\delta_{A W}\left(Y, c_{Y}\right)$ is well defined.

Proposition 4. $\delta_{A W}\left(Y, c_{Y}\right)$ does not depend on the choice of $\left(W, c_{W}\right)$ and hence only depends on the diffeomorphism class of $Y$ and its spin structure $c_{Y}$.

Proof. If we take another $\left(W^{\prime}, c_{W^{\prime}}\right)$, then by the excision property of indices, the difference can be written as

$$
\begin{array}{r}
8 \operatorname{index}_{V}(\mathscr{D} \otimes T)\left((-W) \cup_{Y} W\right)-32 \operatorname{index}_{V} \mathscr{D}\left((-W) \cup_{Y} W\right) \\
-8 \operatorname{index}_{V}(\mathscr{D} \otimes T)\left((-W) \cup_{Y} W^{\prime}\right)+32 \operatorname{index}_{V} \mathscr{D}\left((-W) \cup_{Y} W^{\prime}\right)
\end{array}
$$

$$
-\operatorname{Sign}\left((-W) \cup_{Y} W^{\prime}\right) .
$$

Since $(-W) \cup_{Y} W$ has an orientation-reversing diffeomorphism, the indices of the (twisted) Dirac operators vanish, and by the gravitational anomaly cancellation formula, we have

$8 \operatorname{index}_{V}(\not \otimes T)\left((-W) \cup_{Y} W^{\prime}\right)$

$$
-32 \operatorname{index}_{V} \mathscr{D}\left((-W) \cup_{Y} W^{\prime}\right)+\operatorname{Sign}\left((-W) \cup_{Y} W^{\prime}\right)=0 .
$$


Hence the invariant $\delta_{A W}$ does not depend on the choice of $\left(W, c_{W}\right)$. Because the indices of twisted Dirac operators on closed $V$-manifolds depend only on the diffeomorphism class of the $V$-manifolds and its $V$-spin structures, the signature depends only on the diffeomorphism class and not on the spin structures.

S. Ochanine [1980/81] proved that the signature of every $(8 k+4)$-dimensional spin manifold is always divisible by 16 and hence the following generalized Rohlin invariant is well defined.

Definition 5. Let $\left(Y, c_{Y}\right)$ be a $(8 k+3)$-dimensional spin manifold. Then define $R\left(Y, c_{Y}\right) \equiv \operatorname{Sign}(W)(\bmod 16)$, where $W$ is a $(8 k+4)$-dimensional spin manifold whose spin structure induces $c_{Y}$ on the boundary $Y=\partial W$.

Note that the maximal commuting subalgebra for an irreducible real representation of $C \ell(8 k+4)$ is isomorphic to that of $C \ell(4)$ and is isomorphic to the quaternion $\mathbb{H}$. (See [Lawson and Michelsohn 1989, I., Theorem 5.8].) Hence the Dirac operators $\mathscr{D}$ and $\mathscr{D} \otimes T$ are $\mathbb{H}$-linear, and the $V$-indices index $V \mathscr{D}(Z)$ and index $_{V}(\mathscr{D} \otimes T)(Z)$ are divisible by 2 . Therefore we have the following lemma.

Lemma 6. The invariant $\delta_{A W}\left(Y, c_{Y}\right)$ of 11-dimensional lens spaces $\left(Y, c_{Y}\right)$ is equal to the generalized Rohlin invariant $R\left(Y, c_{Y}\right)$ of $\left(Y, c_{Y}\right)$ modulo 16 , that is, $\delta_{A W}\left(Y, c_{Y}\right) \equiv R\left(Y, c_{Y}\right)(\bmod 16)$.

\section{Spin structures on lens spaces}

We now characterize the set of all spin structures on lens spaces $Y=L(p ; \vec{a})$. Let $X=X(p ; \vec{a})$ be the 12-dimensional spin $V$-manifold associated to the 11dimensional lens space $Y$. Note that $X$ admits an almost $V$-complex structure and hence the canonical $V$-spin ${ }^{c}$ structure $P_{\text {can }}$ on $X$. First we parametrize the set of all $V$-spin ${ }^{c}$ structures on $X$.

Proposition 7. There is a one-to-one correspondence between the set of all $V$ $\operatorname{spin}^{c}$ structures $\operatorname{Spin}_{V}^{c}(X(p ; \vec{a}))$ on $X(p ; \vec{a})$ and the set of all representations $R(\mathbb{Z} / p, U(1)) \cong \mathbb{Z} / p$ from $\mathbb{Z} / p$ to $U(1)$.

Proof. Any $V-\operatorname{spin}^{c}$ structure $P$ on $X$ can be obtained by twisting the canonical $V$-spin ${ }^{c}$ structure $P_{\text {can }}$ by a unique line $V$-bundle $L$ on $X$, and any topological line $V$-bundle $L$ on $X(p ; \vec{a})$ can be obtained as the quotient of the trivial bundle $B^{2 n+2} \times \mathbb{C}$ divided a $\mathbb{Z} / p$ action on $B^{2 n+2} \times \mathbb{C}$ is given by $\zeta_{p}^{l} \cdot(z, u)=\left(\zeta_{p}^{l} z, \rho\left(\zeta_{p}\right)^{l} \cdot u\right)$ for some $U(1)$-representation $\rho: \mathbb{Z} / p \rightarrow U(1)$ of $\mathbb{Z} / p$.

Any $V$-spin structure on $X$ is obtained by twisting the canonical $V$-spin ${ }^{c}$ structure $P_{\text {can }}$ by a half $K^{1 / 2}$ of the canonical line $V$-bundle $K$ of $X$. The canonical line $V$-bundle $K$ corresponds to the representation $a_{1}+a_{2}+\cdots+a_{n} \in \mathbb{Z} / p \cong$ $R(\mathbb{Z} / p, U(1))$, and a half canonical line $V$-bundle corresponds to an element 
$c \in \mathbb{Z} / p \cong R(\mathbb{Z} / p, U(1))$ satisfying $2 c \equiv a_{1}+a_{2}+\cdots+a_{n}(\bmod p)$. Specifically, $X(p ; \vec{a})$ is $V$-spin if and only if $p$ is odd, or $p$ and $a_{1}+\cdots+a_{n}$ are even. If $p$ is odd then the $V$-spin structure $c$ is unique, and if $p$ and $a_{1}+\cdots+a_{n}$ are even then there exist two $V$-spin structures $c$ and $c+p / 2$. Now, a $\operatorname{spin}^{c}$ structure on a manifold $Y$ induces one on $Y \times \mathbb{R}$ and vice versa.

Proposition 8. The restriction map

$$
r: \operatorname{Spin}_{V}^{c}(X(p ; \vec{a})) \ni \sigma \mapsto r(\sigma) \in \operatorname{Spin}^{c}(L(p ; \vec{a}))
$$

is bijective.

Proof. The set $\operatorname{Spin}^{c}(L(p ; \vec{a}))$ of all $\operatorname{spin}^{c}$ structures on $L(p ; \vec{a})$ is in one-toone correspondence with the set $\operatorname{Pic}^{t}(L(p ; \vec{a}))$ of all topological line bundles on $L(p ; \vec{a})$. Likewise, the set $\operatorname{Spin}_{V}^{c}(X(p ; \vec{a}))$ of all $V$-spin ${ }^{c}$ structures on $X(p ; \vec{a})$ is in bijection with the $\operatorname{set}^{\operatorname{Pic}_{V}^{t}}(X(p ; \vec{a}))$ of all topological line $V$-bundles on $X(p ; \vec{a})$. So we must show that the map $i^{*}: \operatorname{Pic}_{V}^{t}(X(p ; \vec{a})) \rightarrow \operatorname{Pic}^{t}(L(p ; \vec{a}))$ is bijective, where $i: L(p ; \vec{a}) \hookrightarrow X(p ; \vec{a})$ is the inclusion. Now any line $V$-bundle in $\operatorname{Pic}_{V}^{t}(X(p ; \vec{a}))$ is by definition the quotient of $B^{2 n} \times \mathbb{C}$ divided by some $\mathbb{Z} / p$ action with representation $\mathbb{Z} / p \rightarrow U(1)$, and hence $\operatorname{Pic}_{V}^{t}(X(p ; \vec{a})) \cong \mathbb{Z} / p$. On the other hand, $\operatorname{Pic}^{t}(L(p ; \vec{a}))$ is isomorphic to $H^{2}(L(p ; \vec{a}) ; \mathbb{Z}) \cong \mathbb{Z} / p$. Now for any $L \in \operatorname{Pic}^{t}(L(p ; \vec{a}))$, the first Chern class $c_{1}(L)$ is a torsion, that is, $c_{1}(L) \in$ $H^{2}(L(p ; \vec{a}) ; \mathbb{Z}) \cong \mathbb{Z} / p$, and by Chern-Weil theory, the de Rham cohomology class $c_{1}(L)=\left[-F_{A} / 2 \pi i\right]$ vanishes for the curvature $F_{A}$ of a connection $A$ on $L$. Therefore we can write $F_{A}=2 \pi i d a$ for some 1 -form $a$ on $L(p ; \vec{a})$. Hence $A-2 \pi i a$ gives a flat connection, and its holonomy $\rho: \mathbb{Z} / p \rightarrow U(1)$ gives an isomorphism of $L$ with the quotient of $S^{2 n} \times \mathbb{C}$ by the $\mathbb{Z} / p$ action given by the holonomy $\rho$. Then the line $V$-bundle $\left(B^{2 n} \times \mathbb{C}\right) /(\mathbb{Z} / p)$ corresponds to $L$, and the surjectivity of $i^{*}$ follows. Since $\operatorname{Pic}^{t}(L(p ; \vec{a}))$ and $\operatorname{Pic}_{V}^{t}(X(p ; \vec{a}))$ are isomorphic to $\mathbb{Z} / p$ and have the same order, the map $i^{*}$ is bijective.

Therefore we can parametrize the set of all $\operatorname{spin}^{c}$ structures on $L(p ; \vec{a})$ by

$$
\mathbb{Z} / p \cong R(\mathbb{Z} / p, U(1)) \cong \operatorname{Spin}_{V}^{c}(X(p ; \vec{a})) \cong \operatorname{Spin}^{c}(L(p ; \vec{a})) .
$$

Proposition 9. Let $\vec{a} \in \mathbb{Z}^{n}$ be an n-tuple of integers coprime to an integer $p$, then the following properties hold.

(1) If $p$ is odd, or $p$ is even and $\sum_{k=1}^{n} a_{k}$ is even, we say the condition (SP) holds; the lens space $L(p ; \vec{a})$ admits a spin structure if and only if (SP) holds.

(2) Suppose (SP) holds for $(p ; \vec{a}) \in \mathbb{Z}^{n+1}$. Then there is a one-to-one correspondence between the set of all spin structures on $L(p ; \vec{a})$ and the set of all elements $c \in \mathbb{Z} / p$ satisfying $2 c=a_{1}+\cdots+a_{n} \in \mathbb{Z} / p$.

Now we assign a sign $\varepsilon \in\{ \pm 1\}$ to the spin structure on $L(p ; \vec{a})$. 
Definition 10. Suppose (SP) holds for $(p, \vec{a}) \in \mathbb{Z}^{n+1}$. For any spin structure $c \in \mathbb{Z} / p$ on $L(p ; \vec{a})$, we define a sign $\varepsilon=\varepsilon(c) \in\{ \pm 1\}$ by

$$
\{ \pm 1\} \ni \varepsilon=\zeta_{p}^{-\left(a_{1}+a_{2}+\cdots+a_{n}\right) / 2} \cdot \zeta_{p}^{c} \in \mathbb{Z} / p \subset U(1) .
$$

Conversely, for each sign $\varepsilon \in\{ \pm 1\}$ we denote by $c(\varepsilon)$ any spin structure $c \in \mathbb{Z} / p$ on $L(p ; \vec{a})$ whose sign is $\varepsilon$.

\section{An explicit formula of the invariant of 11-dimensional lens spaces with spin structures}

This section gives an explicit formula of the invariant $\delta_{A W}$ of spin 11-dimensional lens spaces in terms of trigonometric sums by using the Atiyah-Singer-Kawasaki $V$-index theorem. Let $\left(Y, c_{Y}\right)$ pair 11-dimensional lens space $Y=L(p ; \vec{a})$ and its spin structure $c_{Y}$, and let $\varepsilon$ be the sign of $c_{Y}$.

Theorem 11. Let $L(p ; \vec{a})$ be a 11-dimensional lens space such that $(p ; \vec{a})$ satisfies the condition (SP) in Proposition 9. Then $L(p ; \vec{a})$ admits a spin structure $c$, and let $\varepsilon$ be its sign according to Definition 10. Then

$$
\begin{aligned}
\delta_{A W}(L(p ; \vec{a}) ; c) & =8 \frac{1}{p} \sum_{l=1}^{p-1} \varepsilon^{l} \prod_{j=1}^{n} \frac{1}{\zeta_{p}^{a_{j} l / 2}-\zeta_{p}^{-a_{j} l / 2}}\left(\sum_{j=1}^{n}\left(\zeta_{p}^{a_{j} l}+\zeta_{p}^{-a_{j} l}\right)\right) \\
& -\frac{1}{p} \sum_{l=1}^{p-1} \prod_{j=1}^{n} \frac{\zeta_{p}^{a_{j} l / 2}+\zeta_{p}^{-a_{j} l / 2}}{\zeta_{p}^{a_{j} l / 2}-\zeta_{p}^{-a_{j} l / 2}}-32 \frac{1}{p} \sum_{l=1}^{p-1} \varepsilon^{l} \prod_{j=1}^{n} \frac{1}{\zeta_{p}^{a_{j} l / 2}-\zeta_{p}^{-a_{j} l / 2}} .
\end{aligned}
$$

$\delta_{A W}(\bmod 16)$ gives the generalized Rohlin invariant $R(L(p ; \vec{a}), c)$.

Proof. Let $\left(Y, c_{Y}\right)$ be the corresponding 11-dimensional lens space $L(p ; \vec{a})$ with a spin structure $c_{Y}$ corresponding to $c \in \mathbb{Z} / p$ satisfying $2 c \equiv a_{1}+\cdots+a_{n}(\bmod p)$, where we put $n=6$. Let $\left(X, c_{X}\right)$ be the associated spin $V$-manifold $X(p ; \vec{a})$ with the spin structure $c_{X}$ extending $c_{Y}$. We fix the standard spin structure on $B^{2 n} \subset \mathbb{C}^{n}$. Now the action of $g=\zeta_{p}^{l} \in \mathbb{Z} / p$ on the tangent space $T_{0} B^{n+1}$ is given by $\operatorname{diag}\left(\zeta_{p}^{a_{j} l}\right)_{j=1}^{n}$, and hence the action of $\zeta_{p}^{l}$ on the spin bundle is given by

$$
\operatorname{diag}\left(\prod_{j=1}^{n} \zeta_{p}^{\varepsilon_{j} a_{j} l / 2}\right)_{\varepsilon_{j}= \pm} \cdot \zeta_{p}^{-\left(a_{1}+\cdots+a_{n}\right) / 2} \cdot \zeta_{p}^{c} \in \operatorname{End}(\Delta),
$$

where $\Delta=\mathbb{C}^{2^{n+1}}$ is the fundamental spinor representation and the matrix has $2^{n}$ diagonal entries, one for each set of signs $\left(\varepsilon_{1}, \ldots, \varepsilon_{n}\right)$. We put $\varepsilon=\varepsilon(c)=$ $\zeta_{p}^{-\left(a_{1}+\cdots+a_{n}\right) / 2} \cdot \zeta_{p}^{c} \in\{ \pm 1\} \subset U(1)$ as in Definition 10 . Let $\left(W, c_{W}\right)$ be the $12-$ dimensional smooth spin manifold with boundary $\partial\left(W, c_{W}\right)=-\left(Y, c_{Y}\right)$. Then we can glue $\left(X, c_{X}\right)$ and $\left(W, c_{W}\right)$ along the boundary $\left(Y, c_{Y}\right)$ and obtain the spin 
$V$-manifold $\left(Z, c_{Z}\right)$. Then by the Atiyah-Singer-Kawasaki $V$-index theorem, we have the equalities

$$
\begin{gathered}
\operatorname{index}_{V} \mathscr{D}(Z)=\hat{A}(Z)+\frac{1}{p} \sum_{l=1}^{p-1} \varepsilon^{l} \prod_{j=1}^{n} \frac{1}{\zeta_{p}^{a_{j} l / 2}-\zeta_{p}^{-a_{j} l / 2}}, \\
\operatorname{index}_{V} \mathscr{D}(Z) \otimes T=\hat{A}(Z) \operatorname{ch}(T)+\frac{1}{p} \sum_{l=1}^{n} \varepsilon^{l} \frac{\sum_{j=1}^{n}\left(\zeta_{p}^{a_{j} l}+\zeta_{p}^{a_{j} l}\right)}{\prod_{j=1}^{n}\left(\zeta_{p}^{a_{j} l / 2}-\zeta_{p}^{-a_{j} l / 2}\right)}, \\
\operatorname{Sign}(Z)=L(Z)+\frac{1}{p} \sum_{l=1}^{p-1} \prod_{j=1}^{n} \frac{\zeta_{p}^{a_{j} l / 2}+\zeta_{p}^{-a_{j} l / 2}}{\zeta_{p}^{a_{j} l / 2}-\zeta_{p}^{-a_{j} l / 2}}
\end{gathered}
$$

Then we can compute the $\delta_{A W}$-invariant as follows.

$$
\begin{aligned}
& \delta_{A W}\left(\left(Y, c_{Y}\right),\left(X, c_{X}\right)\right) \\
& =8\left(\hat{A}\left(X \cup_{Y} W\right)+\frac{1}{p} \sum_{l=1}^{p-1} \varepsilon^{l} \frac{\sum_{j=1}^{n}\left(\zeta_{p}^{a_{j} l}+\zeta_{p}^{-a_{j} l}\right)}{\prod_{j=1}^{n}\left(\zeta_{p}^{a_{j} l / 2}-\zeta_{p}^{-a_{j} l / 2}\right)}\right) \\
& -32\left(\hat{A}\left(X \cup_{Y} W\right) \cdot \operatorname{ch}\left(T\left(X \cup_{Y} W\right)\right)+\frac{1}{p} \sum_{l=1}^{p-1} \varepsilon^{l} \prod_{j=1}^{n} \frac{1}{\zeta_{p}^{a_{j} l / 2}-\zeta_{p}^{-a_{j} l / 2}}\right)-\operatorname{Sign}(W) \\
& =8 \hat{A}(Z)-32 \hat{A}(Z) \operatorname{ch}(T(Z))-\operatorname{Sign}(W) \\
& \quad+8 \frac{1}{p} \sum_{l=1}^{p-1} \varepsilon^{l} \frac{\sum_{j=1}^{n}\left(\zeta_{p}^{a_{j} l}+\zeta_{p}^{-a_{j} l}\right)}{\prod_{j=1}^{n}\left(\zeta_{p}^{a_{j} l / 2}-\zeta_{p}^{-a_{j} l / 2}\right)}-32 \frac{1}{p} \sum_{l=1}^{p-1} \varepsilon^{l} \prod_{j=1}^{n} \frac{1}{\zeta_{p}^{a_{j} l / 2}-\zeta_{p}^{-a_{j} l / 2}} .
\end{aligned}
$$

Now for the first term, by the gravitational anomaly cancellation formula, we have

$$
\begin{aligned}
& 8 \hat{A}(Z)-32 \hat{A}(Z) \operatorname{ch}(T(Z))-\operatorname{Sign}(W)=L(Z)-\operatorname{Sign}(W) \\
& =\operatorname{Sign}(Z)-\frac{1}{p} \sum_{l=1}^{p-1} \prod_{j=1}^{n} \frac{\zeta_{p}^{a_{j} l / 2}+\zeta_{p}^{-a_{j} l / 2}}{\zeta_{p}^{a_{j} l / 2}-\zeta_{p}^{-a_{j} l / 2}}-\operatorname{Sign}(W) \\
& =\operatorname{Sign}(X)+\operatorname{Sign}(W)-\frac{1}{p} \sum_{l=1}^{p-1} \prod_{j=1}^{n} \frac{\zeta_{p}^{a_{j} l / 2}+\zeta_{p}^{-a_{j} l / 2}}{\zeta_{p}^{a_{j} l / 2}-\zeta_{p}^{-a_{j} l / 2}}-\operatorname{Sign}(W) \\
& =\operatorname{Sign}(X)-\frac{1}{p} \sum_{l=1}^{p-1} \prod_{j=1}^{n} \frac{\zeta_{p}^{a_{j} l / 2}+\zeta_{p}^{-a_{j} l / 2}}{\zeta_{p}^{a_{j} l / 2}-\zeta_{p}^{-a_{j} l / 2}}=-\frac{1}{p} \sum_{l=1}^{p-1} \prod_{j=1}^{n} \frac{\zeta_{p}^{a_{j} l / 2}+\zeta_{p}^{-a_{j} l / 2}}{\zeta_{p}^{a_{j} l / 2}-\zeta_{p}^{-a_{j} l / 2}} . \square
\end{aligned}
$$

Notation 12. We denote $\delta_{A W}(L(p ; \vec{a}) ; c)$ by $\delta_{A W}\left(p ; a_{1}, \ldots, a_{6} ; \varepsilon\right)$ in the sequel. 
As an application of the explicit formula of the generalized Rohlin invariant of lens spaces by using $\delta_{A W}$, we have the following theorem. Let $S^{2 n-1}$ be the standard sphere. We say a $\mathbb{Z} / p$ action on $S^{2 n-1}$ is of type $\left(p ; a_{1}, \ldots, a_{n} ; \varepsilon\right)$ if and only if $\mathbb{Z} / p$ action lifts to the spin structure on $S^{2 n-1}$ so that the quotient $S^{2 n-1} /(\mathbb{Z} / p)$ is diffeomorphic to the lens space $L\left(p ; a_{1}, \ldots, a_{n}\right)$ with spin structure $c(\varepsilon)$. Let $\delta_{\text {Sign }}\left(p ; a_{1}, \ldots, a_{n}\right)$ be the higher-dimensional Dedekind sum (localization term of the $V$-index of the signature operator)

$$
\delta_{\text {Sign }}\left(p ; a_{1}, \ldots, a_{n}\right)=\frac{1}{p} \sum_{l=1}^{p-1} \prod_{j=1}^{n} \frac{\zeta_{p}^{a_{j} l / 2}+\zeta_{p}^{-a_{j} l / 2}}{\zeta_{p}^{a_{j} l / 2}-\zeta_{p}^{-a_{j} l / 2}},
$$

Set $\tilde{\delta}_{A W+\operatorname{Sign}}\left(p ; a_{1}, \ldots, a_{n} ; \varepsilon\right)=p\left(\delta_{A W}\left(p ; a_{1}, \ldots, a_{6} ; \varepsilon\right)+\delta_{\operatorname{Sign}}\left(p ; a_{1}, \ldots, a_{6}\right)\right)$.

Theorem 13. Let $S^{11}$ be the standard sphere of dimension 11 with a free $\mathbb{Z} / p$ action of type $\left(p ; a_{1}, \ldots, a_{n} ; \varepsilon\right)$. If $\widetilde{W}$ is a 12-dimensional spin manifold with boundary $\partial \widetilde{W} \cong S^{11}$ with a free $\mathbb{Z} / p$ action preserving the spin structure that extends that on $S^{11}$. Then $\tilde{\delta}_{A W+\operatorname{Sign}}\left(p ; a_{1}, \ldots, a_{6} ; \varepsilon\right)$ must be an integer and the signature $\operatorname{Sign}(\widetilde{W})$ of $\widetilde{W}$ satisfies

$$
\operatorname{Sign}(\widetilde{W}) \equiv \tilde{\delta}_{A W+\operatorname{Sign}}\left(p ; a_{1}, \ldots, a_{6} ; \varepsilon\right) \quad(\bmod 16 p) .
$$

Proof. Suppose such a 12-dimensional spin manifold $\widetilde{W}$ with a $\mathbb{Z} / p$ action exists. Then we divide $\widetilde{W}$ by the $\mathbb{Z} / p$ action to obtain a 12 -dimensional spin manifold $W=\widetilde{W} /(\mathbb{Z} / p)$ with boundary $\partial W \cong L\left(p ; a_{1}, \ldots, a_{6}\right)$ and spin structure $c(\varepsilon)$. By Lemma 6 the signature of $W$ is equal to $\delta_{A W}\left(p ; a_{1}, \ldots, a_{6} ; \varepsilon\right)$ modulo 16 . On the other hand, by the $G$-signature formula

$$
\operatorname{Sign}(W)=\frac{1}{p} \operatorname{Sign}(\widetilde{W})-\delta_{\operatorname{Sign}}\left(p ; a_{1}, \ldots, a_{6}\right),
$$

we have $\delta_{A W}\left(p ; a_{1}, \ldots, a_{6} ; \varepsilon\right) \equiv(1 / p) \operatorname{Sign}(\widetilde{W})-\delta_{\operatorname{Sign}}\left(p ; a_{1}, \ldots, a_{6}\right)(\bmod 16)$. Hence the assertion follows.

\section{Examples}

We next give several examples of the invariants $\delta_{A W}$ and $R$ for 11-dimensional lens spaces with spin structures $c(\varepsilon)$ parametrized by $\varepsilon \in\{ \pm 1\}$. We calculate also the $\alpha$-invariant of W. Browder and G. Livesay [1967] for differentiable, fixed-pointfree involutions on homotopy spheres according to an explicit calculation, by F. Hirzebruch and D. Zagier [1974], for lens spaces $L\left(p ; a_{1}, \ldots, a_{n}\right)$ in which all the $a_{i}$ are odd. We also calculate $\tilde{\delta}_{A W+\operatorname{Sign}}(\varepsilon)(\bmod 16 p)$ which determines $\operatorname{Sign}(\widetilde{W})$ modulo $16 p$ of the 12 -dimensional spin manifold $\widetilde{W}$ with a $\mathbb{Z} / p$ action, boundary $S^{11}$, and a $\mathbb{Z} / p$ action of type $\left(p ; a_{1}, \ldots, a_{n} ; \varepsilon\right)$. Note that the torsion invariant of K. Reidemeister [1938] and W. Franz [1938] gives a complete classification of 
diffeomorphism types of lens spaces. In fact, each lens space is diffeomorphic to a unique lens space of the form $\pm L\left(p ; a_{1}, \ldots, a_{n}\right)$ with $1 \leq a_{1} \leq \cdots \leq a_{n}<p / 2$. By results of M. Rueff [1939] and P. Olum [1953], two lens spaces $L\left(p ; a_{1}, \ldots, a_{n}\right)$ and $L\left(p ; a_{1}^{\prime}, \ldots, a_{n}^{\prime}\right)$ are homotopy equivalent if and only if

$$
a_{1}^{\prime} \cdots a_{n}^{\prime} \equiv \pm k^{n} a_{1} \cdots a_{n} \quad(\bmod p) .
$$

for some integer $k$. In the following examples, we compare behaviors of these invariants for each lens space homotopy type. These classical results concerning the torsion of J. H. C. Whitehead are surveyed by J. Milnor [1966].

Example 14. The lens spaces $L(p ; 1,1,1,1,1,1)$ for $2 \leq p \leq 20$.

\begin{tabular}{lllll}
\hline$p$ & $\delta_{A W}(\varepsilon)$ & $R(\varepsilon)$ & $\alpha$ & $\tilde{\delta}_{A W+\operatorname{Sign}}(\varepsilon)(\bmod 16 p)$ \\
\hline 2 & $1(+1),-1(-1)$ & $1(+1), 15(-1)$ & 1 & $2(+1),-2(-1)$ \\
3 & $2(+1)$ & $2(+1)$ & 18 & $\frac{160}{27}(+1)$ \\
4 & $3(+1),-1(-1)$ & $3(+1), 15(-1)$ & 99 & $10(+1),-6(-1)$ \\
5 & $4(+1)$ & $4(+1)$ & 340 & $\frac{32}{5}(+1)$ \\
6 & $5(+1), 15(-1)$ & $5(+1), 15(-1)$ & 901 & $-\frac{650}{27}(+1), \frac{970}{27}(-1)$ \\
7 & $6(+1)$ & $6(+1)$ & 2022 & $-\frac{48}{7}(+1)$ \\
8 & $7(+1), 95(-1)$ & $7(+1), 15(-1)$ & 4039 & $42(+1),-22(-1)$ \\
9 & $8(+1)$ & $8(+1)$ & 7400 & $\frac{1888}{27}(+1)$ \\
10 & $9(+1), 335(-1)$ & $9(+1), 15(-1)$ & 12681 & $-\frac{118}{5}(+1), \frac{182}{5}(-1)$ \\
11 & $10(+1)$ & $10(+1)$ & 20602 & $-48(+1)$ \\
12 & $11(+1), 895(-1)$ & $11(+1), 15(-1)$ & 32043 & $\frac{1294}{27}(+1), \frac{2590}{27}(-1)$ \\
13 & $12(+1)$ & $12(+1)$ & 48060 & $80(+1)$ \\
14 & $13(+1), 2015(-1)$ & $13(+1), 15(-1)$ & 69901 & $-\frac{146}{7}(+1), \frac{50}{7}(-1)$ \\
15 & $14(+1)$ & $14(+1)$ & 99022 & $\frac{12464}{135}(+1)$ \\
16 & $15(+1), 4031(-1)$ & $15(+1), 15(+1)$ & 137103 & $42(+1), 42(-1)$ \\
17 & $16(+1)$ & $0(+1)$ & 186064 & $96(+1)$ \\
18 & $17(+1), 7391(-1)$ & $1(+1), 15(-1)$ & 248081 & $-\frac{1514}{27}(+1),-\frac{2486}{27}(-1)$ \\
19 & $18(+1)$ & $2(+1)$ & 325602 & $32(+1)$ \\
20 & $19(+1), 12671(-1)$ & $3(+1), 15(-1)$ & 421363 & $-\frac{718}{5}(+1), \frac{482}{5}(-1)$ \\
\hline
\end{tabular}

(1) In fact, this computation for $2 \leq p \leq 20$ agrees with a calculation by S. Ochanine [1980/81] of the generalized Rohlin invariant of $(8 k+3)$-dimensional lens spaces of type $L(p ; 1, \ldots, 1)$ for any integer $p$.

(2) The Browder-Livesay invariant $\alpha$ distinguishes all diffeomorphism classes of lens spaces of type $L(p ; 1,1,1,1,1,1)$ for $2 \leq p \leq 20$. 
(3) By Theorem 13, we see for example that the sphere $S^{11}$ with $\mathbb{Z} / 4$ action of type $(4 ; 1,1,1,1,1,1 ;+1)$ cannot be the boundary of a 12 -dimensional spin manifold $W$ with a $\mathbb{Z} / 4$ action that extends the action on $S^{11}$ to one preserving the spin structure such that $\operatorname{Sign}(\widetilde{W}) \not \equiv 10(\bmod 16 \cdot 4)$. Similarly, considering the same type, but with negative $\varepsilon$, the same statement holds, but with $\operatorname{Sign}(\widetilde{W}) \not \equiv 10(\bmod 16 \cdot 4)$ replaced by $\operatorname{Sign}(\widetilde{W}) \not \equiv-6(\bmod 16 \cdot 4)$. On the other hand, since $\tilde{\delta}_{A W+\operatorname{Sign}}(10 ; 1,1,1,1,1,1 ;+1) \equiv-118 / 5(\bmod 160)$ and $\tilde{\delta}_{A W+\operatorname{Sign}}(10 ; 1,1,1,1,1,1 ;-1) \equiv 118 / 5(\bmod 160)$ are not integers, we see from Theorem 13 that the sphere $S^{11}$ with a $\mathbb{Z} / 10$ action of type $(10 ; 1,1,1,1,1,1 ; \pm 1)$ cannot be the boundary of any 12-dimensional spin manifold $W$ whose $\mathbb{Z} / 10$ action is a spin-structure-preserving extension of the action on $S^{11}$.

Example 15. The lens spaces $L\left(8 ; a_{1}, \ldots, a_{6}\right)$ for $1 \leq a_{1} \leq \cdots \leq a_{6} \leq 3$.

\begin{tabular}{lllll}
\hline$\left(a_{1}, \ldots, a_{6}\right)$ & $\delta_{A W}(\varepsilon)$ & $R(\varepsilon)$ & $\alpha$ & $\tilde{\delta}_{A W+\operatorname{Sign}}(\varepsilon)(\bmod 16 \cdot 8)$ \\
\hline$(1,1,1,1,1,1)$ & $7(+1), 95(-1)$ & $7(+1), 15(-1)$ & 4039 & $42(+1),-22(-1)$ \\
$(1,1,1,1,3,3)$ & $7(+1),-1(-1)$ & $7(+1), 15(-1)$ & 359 & $42(+1),-22(-1)$ \\
$(1,1,3,3,3,3)$ & $7(+1),-1(-1)$ & $7(+1), 15(-1)$ & 103 & $42(+1),-22(-1)$ \\
$(3,3,3,3,3,3)$ & $7(+1), 95(-1)$ & $7(+1), 15(-1)$ & 4039 & $42(+1),-22(-1)$ \\
\hline$(1,1,1,1,1,3)$ & $1(+1), 17(-1)$ & $1(+1), 1(-1)$ & 1201 & $-58(+1),-58(-1)$ \\
$(1,1,1,3,3,3)$ & $1(+1), 1(-1)$ & $1(+1), 1(-1)$ & 97 & $6(+1), 6(-1)$ \\
$(1,3,3,3,3,3)$ & $17(+1), 1(-1)$ & $1(+1), 1(-1)$ & -527 & $-58(+1),-58(-1)$ \\
\hline
\end{tabular}

(1) Note that in this example, there are two homotopy equivalence classes, one given by the first four rows and the other given by the remaining three. The generalized Rohlin invariants precisely detect these classes and also detect spin structures in the first class. On the other hand, the $\delta_{A W}$-invariants do not necessarily take the same value within each homotopy equivalence class; for example, $\delta_{A W}(\varepsilon)$ takes different values in the table's first and second rows. Hence then $\delta_{A W}$-invariant detects the difference between the diffeomorphism type of $L(8 ; 1,1,1,1,1,1)$ and that of $L(8 ; 1,1,1,1,3,3)$.

(2) The Browder-Livesay invariant $\alpha$ distinguishes diffeomorphism classes of lens spaces of type $L\left(8 ; a_{1}, \ldots, a_{6}\right)$ except for the first and fourth row.

(3) By Theorem 13, we see for example that the sphere $S^{11}$ with a $\mathbb{Z} / 8$ action of type $(8 ; 1,1,1,1,3,3 ;+1)$ cannot be the boundary of a 12-dimensional spin manifold $\widetilde{W}$ with a $\mathbb{Z} / 8$ action that extends the action on $S^{11}$ to one preserving the spin structure such that $\operatorname{Sign}(\widetilde{W}) \not \equiv 42(\bmod 128)$. Likewise, considering the same action, but with negative $\varepsilon$, the same statement holds, but with $\operatorname{Sign}(\widetilde{W}) \not \equiv-22(\bmod 128)$ instead. 
Example 16. The lens spaces $L\left(9 ; a_{1}, \ldots, a_{6}\right)$ for $1 \leq a_{1} \leq \cdots \leq a_{6} \leq 4$.

\begin{tabular}{lllll}
\hline$\left(9 ; a_{1}, \ldots, a_{6}\right)$ & $\delta_{A W}(\varepsilon)$ & $R(\varepsilon)$ & $\alpha$ & $\tilde{\delta}_{A W+\operatorname{Sign}}(\varepsilon)(\bmod 16 \cdot 9)$ \\
\hline$(9 ; 1,1,1,1,1,1)$ & $8(+1)$ & $8(+1)$ & 7400 & $(1888 / 27)(+1)$ \\
$(9 ; 1,1,1,1,2,4)$ & $0(+1)$ & $0(+1)$ & - & $-(592 / 27)(+1)$ \\
$(9 ; 1,1,1,2,2,2)$ & $16(-1)$ & $0(-1)$ & - & $-(1888 / 27)(-1)$ \\
$(9 ; 1,1,1,4,4,4)$ & $-16(-1)$ & $0(-1)$ & - & $(1888 / 27)(-1)$ \\
$(9 ; 1,1,2,2,4,4)$ & $8(+1)$ & $8(+1)-$ & $(1888 / 27)(+1)$ \\
$(9 ; 1,2,2,2,2,4)$ & $0(-1)$ & $0(-1)$ & - & $(592 / 27)(-1)$ \\
$(9 ; 1,2,4,4,4,4)$ & $0(-1)$ & $0(-1)$ & - & $-(592 / 27)(-1)$ \\
$(9 ; 2,2,2,2,2,2)$ & $8(+1)$ & $8(+1)-$ & $(1888 / 27)(+1)$ \\
$(9 ; 2,2,2,4,4,4)$ & $16(+1)$ & $0(+1)$ & - & $-(1888 / 27)(+1)$ \\
$(9 ; 4,4,4,4,4,4)$ & $8(+1)$ & $8(+1)$ & - & $(1888 / 27)(+1)$ \\
\hline$(9 ; 1,1,1,1,1,2)$ & $80(-1)$ & $0(-1)$ & - & $(1568 / 27)(-1)$ \\
$(9 ; 1,1,1,1,4,4)$ & $8(+1)$ & $8(+1)$ & - & $(1024 / 27)(+1)$ \\
$(9 ; 1,1,1,2,2,4)$ & $0(-1)$ & $0(-1)$ & - & $-(272 / 27)(-1)$ \\
$(9 ; 1,1,2,2,2,2)$ & $8(+1)$ & $8(+1)$ & - & $(1024 / 27)(+1)$ \\
$(9 ; 1,1,2,4,4,4)$ & $0(+1)$ & $0(+1)$ & - & $(272 / 27)(+1)$ \\
$(9 ; 1,2,2,2,4,4)$ & $0(-1)$ & $0(-1)$ & - & $(272 / 27)(-1)$ \\
$(9 ; 1,4,4,4,4,4)$ & $-80(-1)$ & $0(-1)$ & - & $-(1568 / 27)(-1)$ \\
$(9 ; 2,2,2,2,2,4)$ & $80(+1)$ & $0(+1)$ & - & $(1568 / 27)(+1)$ \\
$(9 ; 2,2,4,4,4,4)$ & $8(+1)$ & $8(+1)$ & - & $(1024 / 27)(+1)$ \\
\hline$(9 ; 1,1,1,1,1,4)$ & $16(-1)$ & $0(-1)$ & - & $-(1136 / 27)(-1)$ \\
$(9 ; 1,1,1,1,2,2)$ & $8(+1)$ & $8(+1)$ & - & $(1456 / 27)(+1)$ \\
$(9 ; 1,1,1,2,4,4)$ & $0(-1)$ & $0(-1)$ & - & $-(160 / 27)(-1)$ \\
$(9 ; 1,1,2,2,2,4)$ & $0(+1)$ & $0(+1)$ & - & $-(160 / 27)(+1)$ \\
$(9 ; 1,1,4,4,4,4)$ & $8(+1)$ & $8(+1)-$ & $(1456 / 27)(+1)$ \\
$(9 ; 1,2,2,2,2,2)$ & $-16(-1)$ & $0(-1)$ & - & $(1136 / 27)(-1)$ \\
$(9 ; 1,2,2,4,4,4)$ & $0(-1)$ & $0(-1)$ & - & $(160 / 27)(-1)$ \\
$(9 ; 2,2,2,2,4,4)$ & $8(+1)$ & $8(+1)-$ & $(1456 / 27)(+1)$ \\
$(9 ; 2,4,4,4,4,4)$ & $-16(+1)$ & $0(+1)-$ & $(1136 / 27)(+1)$ \\
\hline & & & & \\
\hline
\end{tabular}

(1) Note that in this example $L\left(9 ; a_{1}, \ldots, a_{6}\right)$ with $1 \leq a_{1} \leq \cdots \leq a_{6} \leq 4$, there are three homotopy equivalence classes, which are grouped together as before within horizontal lines. In this case, both invariants do not necessarily detect homotopy equivalent classes. For example, the lens spaces 
$L(9 ; 1,1,1,1,1,1), L(9 ; 1,1,1,1,4,4)$, and $L(9 ; 1,1,1,1,2,2)$ belong to different homotopy equivalence classes, but all three have $\delta_{A W}=8$. There are a number of lens spaces for which both $\delta_{A W}(\varepsilon)$ and $R(\varepsilon)$ vanish. At the same times, there are entries in which $R(\varepsilon)$ vanishes, but $\delta_{A W}(\varepsilon)$ does not. For example, the $\delta_{A W}$-invariants of lens spaces $L(9 ; 1,1,1,1,2,4)$ and $L(9 ; 1,1,1,2,2,2)$, which live in the same homotopy equivalence class and have vanishing Rohlin invariant, are 0 and 16, respectively. Hence, they are not diffeomorphic. The author does not know if they are homeomorphic.

(2) The table gives the Browder-Livesay invariant only for $L(9 ; 1,1,1,1,1,1)$, since there is apparently no natural involution for other types containing some even $a_{i}$.

(3) None of the $\tilde{\delta}_{A W+\operatorname{Sign}}(\varepsilon)$ values are integers. Hence by Theorem 13, we see that the sphere $S^{11}$ with $\mathbb{Z} / p$ action of type $\left(9 ; a_{1}, \ldots, a_{6} ;+1\right)$ cannot be the boundary of a 12 -dimensional spin manifold $\widetilde{W}$ whose $\mathbb{Z} / p$ action is a spinstructure-preserving extension of the action on $S^{11}$.

\section{A reciprocity property of the invariant}

Next, we prove a reciprocity property of the invariant $\delta_{A W}$ of 11-dimensional lens spaces with spin structures. In particular, this equality gives a simple reciprocity property of the generalized Rohlin invariant of 11-dimensional lens spaces with spin structures modulo 16.

Theorem 17. Let $\left(a_{0}, \ldots, a_{6}\right)$ be pairwise coprime integers satisfying $\sum_{k=0}^{6} a_{k} \equiv 0$ (mod 2). Then, letting $\alpha:=\sum_{k=0}^{6} a_{k}$, we have

$$
\begin{aligned}
& \sum_{k=0}^{6} \delta_{A W}\left(a_{k} ; a_{1}, \ldots, \check{a}_{k}, \ldots, a_{6} ;-1\right) \\
& \quad=-1+16 \cdot \sum_{h:-\alpha / 2+a_{h} \geq 0} \sharp\left\{\left(k_{0}, \ldots, k_{6}\right) \in \mathbb{Z}_{\geq 0}^{7} \mid k_{0} a_{0}+\cdots+k_{6} a_{6}=-\alpha / 2+a_{h}\right\} .
\end{aligned}
$$

Remark 18. This reciprocity law gives a simple reciprocity for the generalized Rohlin invariant for the 11-dimensional lens spaces with spin structures modulo 16, namely $\sum_{k=0}^{6} R\left(L\left(a_{k} ; a_{1}, \ldots, \check{a}_{k}, \ldots, a_{6}\right), c(-1)\right) \equiv-1(\bmod 16)$. This equality can be proved directly from the Novikov additivity of the signatures.

Remark 19. Now consider the integer-valued invariant $\sigma(p ; a, b, \varepsilon)$ of the 3dimensional lens space $L(p ; a, b)$ with a spin structure that is a singular point contribution of the $w$-invariant [Fukumoto et al. 2001; Fukumoto 2002]. This invariant satisfies that reciprocity property that

$$
\sigma(p ; q, r,-1)+\sigma(q ; r, p,-1)+\sigma(r ; p, q,-1)=-1
$$


for pairwise coprime integers $p, q, r$ satisfying $p+q+r \equiv 0(\bmod 2)$. This property is essentially the same as a reciprocity property of an arithmetic sum $S(a, c)$, given by B. Berndt [1978], concerning the transformation formula of the logarithm of the classical theta function; in fact $S(h, k)=1-\sigma(k ; 1, h,-1)$ [Fukumoto et al. 2001; Fukumoto 2002]. Theorem 17 is an 11-dimensional version of this statement.

Proof. We put $n=6$ in the following argument. Consider the rational function $f(z)$ on $\mathbb{C}$ given by

$$
f(z):=\frac{1}{z} \cdot \frac{z^{-\sum_{k=0}^{n} a_{k} / 2} \cdot \sum_{h=0}^{n}\left(z^{a_{h}}+z^{-a_{h}}\right)}{\prod_{k=0}^{n}\left(1-z^{-a_{k}}\right)}=\sum_{h=0}^{n}\left(g_{-\alpha / 2+a_{h}}(z)+g_{-\alpha / 2-a_{h}}(z)\right),
$$

where $g_{m}(z)$ is the rational function

$$
g_{m}(z):=\frac{1}{z} \prod_{k=0}^{n} \frac{1}{1-z^{-a_{k}}} \cdot z^{m}
$$

First we consider the residues of $g_{m}(z) d z \cdot g_{m}(z)$ has poles $z=\zeta_{k}^{l}$ for $l=0,1, \ldots, a_{k}$ and $k=0, \ldots, n$ and has poles at $z=0$ or $z=\infty$, depending on the multiplicities $\vec{a}$. The residue at $z=\zeta_{k}^{l}$ is

$$
\operatorname{res}_{z=\zeta_{k}^{l}} g_{m}(z) d z=\frac{1}{a_{k}} \prod_{j \neq k} \frac{1}{1-\zeta_{k}^{-a_{j} l}} \cdot \zeta_{k}^{m l}
$$

for $l=1, \ldots, a_{k}$ and $k=0, \ldots, n$, and

$$
\operatorname{res}_{z=1} g_{m}(z) d z=\frac{1}{a_{0} \cdots a_{n}} \operatorname{res}_{t=0}\left(\prod_{k=0}^{n} \frac{a_{k} t}{1-e^{-a_{k} t}} \cdot e^{m t} \cdot \frac{d t}{t^{n}}\right) .
$$

The origin $z=0$ is a pole if $m \leq-\sum_{k=0}^{n} a_{k}=-\alpha$, and its residue is

$$
\operatorname{res}_{z=0} g_{m}(z) d z=(-1)^{n+1} \sharp\left\{\left(k_{0}, \ldots, k_{n}\right) \in \mathbb{Z}_{\geq 0}^{n+1} \mid k_{0} a_{0}+\cdots+k_{n} a_{n}=-\alpha-m\right\} \text {. }
$$

The infinity $z=\infty$ is a pole if $m \geq 0$, and its residue is

$$
\operatorname{res}_{z=\infty} g_{m}(z) d z=-\sharp\left\{\left(k_{0}, \ldots, k_{n}\right) \in \mathbb{Z}_{\geq 0}^{n+1} \mid k_{0} a_{0}+\cdots+k_{n} a_{n}=m\right\} .
$$

Note that $\alpha / 2+a_{h}>0$ always holds, and hence $-\alpha / 2+a_{h}>-\alpha$ or, equivalently, $-\alpha / 2-a_{h}<0$. On the other hand, $-\alpha / 2+a_{h} \geq 0$ if and only if $-\alpha / 2-a_{h} \leq-\alpha$. Now, supposing $-\alpha / 2+a_{h} \geq 0$, we have

$$
\begin{aligned}
& \operatorname{res}_{z=\infty} g_{-\alpha / 2+a_{h}}(z) d z=-\sharp\left\{\left(k_{0}, \ldots, k_{n}\right) \in \mathbb{Z}_{\geq 0}^{n+1} \mid \sum_{i=0}^{n} k_{i} a_{i}=-\alpha / 2+a_{h}\right\}, \\
& \operatorname{res}_{z=0} g_{-\alpha / 2+a_{h}}(z) d z=0, \\
& \operatorname{res}_{z=\infty} g_{-\alpha / 2-a_{h}}(z) d z=0, \\
& \operatorname{res}_{z=0} g_{-\alpha / 2-a_{h}}(z) d z=(-1)^{n+1} \sharp\left\{\left(k_{0}, \ldots, k_{n}\right) \in \mathbb{Z}_{\geq 0}^{n+1} \mid \sum_{i=0}^{n} k_{i} a_{i}=-\alpha / 2+a_{h}\right\} .
\end{aligned}
$$


On the other hand if $(-\alpha<)-\alpha / 2+a_{h}<0$ then $-\alpha / 2-a_{h}>-\alpha$, and hence the residues of $g_{-\alpha / 2+a_{h}}(z)$ and $g_{-\alpha / 2-a_{h}}(z)$ at $z=0, \infty$ all vanish. Then by the residue theorem, we have the equality

$$
\begin{aligned}
0= & \sum_{t \in \mathbb{C}} \operatorname{res}_{z=t} f(z) d t=\sum_{h=0}^{n} \sum_{t \in \mathbb{C}} \operatorname{res}_{z=t}\left(g_{-\alpha / 2+a_{h}}(z) d z+g_{-\alpha / 2-a_{h}}(z) d z\right) \\
= & \sum_{k=0}^{n} \sum_{l=1}^{a_{k}-1} \frac{1}{a_{k}} \prod_{j \neq k} \frac{1}{1-\zeta_{k}^{-a_{j} l}} \cdot \zeta_{k}^{(-\alpha / 2) l} \cdot \sum_{h=0}^{n}\left(\zeta_{k}^{a_{h} l}+\zeta_{k}^{-a_{h} l}\right) \\
& +\frac{1}{a_{0} \cdots a_{n}} \sum_{h=0}^{n}\left(T_{n}^{\left(-\alpha / 2+a_{h}\right)}\left(c_{1}, \ldots, c_{n}\right)+T_{n}^{\left(-\alpha / 2-a_{h}\right)}\left(c_{1}, \ldots, c_{n}\right)\right) \\
& -\sum_{h:-\alpha / 2+a_{h} \geq 0}\left(1+(-1)^{n}\right) \sharp\left\{\left(k_{0}, \ldots, k_{n}\right) \in \mathbb{Z}_{\geq 0}^{n+1} \mid \sum_{i=0}^{n} k_{i} a_{i}=-\alpha / 2+a_{h}\right\} .
\end{aligned}
$$

Here the second equality uses a formula from the proof of [Fukumoto 2005, Theorem 1]. Rewriting $\sum_{h=0}^{n}\left(\zeta_{k}^{a_{h} l}+\zeta_{k}^{-a_{h} l}\right)=\sum_{h \neq k}\left(\zeta_{k}^{a_{h} l}+\zeta_{k}^{-a_{h} l}\right)+2$ and using the reciprocity law corresponding to the spin Dirac operator, that is,

$$
\sum_{k=0}^{n} \sum_{l=1}^{a_{k}-1} \frac{1}{a_{k}} \prod_{j \neq k} \frac{1}{1-\zeta_{k}^{-a_{j} l}} \cdot \zeta_{k}^{(-\alpha / 2) l}=-\frac{1}{a_{0} \cdots a_{n}} \operatorname{res}_{t=0}\left(\prod_{k=0}^{n} \frac{a_{k} t}{e^{a_{k} t / 2}-e^{-a_{k} t / 2}} \cdot \frac{d t}{t^{n}}\right),
$$

we obtain the reciprocity law corresponding to the Dirac operator twisted by the tangent $V$-bundle:

$$
\begin{aligned}
& \sum_{k=0}^{n} \sum_{l=1}^{a_{k}-1} \frac{1}{a_{k}}(-1)^{l} \prod_{j \neq k} \frac{1}{\zeta_{k}^{a_{j} l / 2}-\zeta_{k}^{-a_{j} l / 2}} \cdot \sum_{h \neq k}\left(\zeta_{k}^{a_{h} l}+\zeta_{k}^{-a_{h} l}\right) \\
& =-\frac{1}{a_{0} \cdots a_{n}} \operatorname{res}_{t=0}\left(\prod_{k=0}^{n} \frac{a_{k} t}{e^{a_{k} t / 2}-e^{-a_{k} t / 2}} \cdot\left(\sum_{h=0}^{n}\left(e^{a_{h} t}+e^{-a_{h} t}\right)-2\right) \cdot \frac{d t}{t^{n}}\right) \\
& \quad+\left(1+(-1)^{n}\right) \sum_{h ;-\alpha / 2+a_{h} \geq 0} \sharp\left\{\left(k_{0}, \ldots, k_{n}\right) \in \mathbb{Z}_{\geq 0}^{n+1} \mid \sum_{i=0}^{n} k_{i} a_{i}=-\alpha / 2+a_{h}\right\} .
\end{aligned}
$$

Then applying the reciprocity law corresponding to the signature operator, that is,

$$
\sum_{k=0}^{n} \frac{1}{a_{k}} \sum_{l=1}^{a_{k}-1} \prod_{j \neq k} \frac{\zeta_{k}^{a_{j} l / 2}+\zeta_{k}^{-a_{j} l / 2}}{\zeta_{k}^{a_{j} l / 2}-\zeta_{k}^{-a_{j} l / 2}}=1-\frac{1}{a_{0} \cdots a_{n}} \operatorname{res}_{t=0}\left(\prod_{k=0}^{n} a_{k} t \cdot \frac{e^{a_{k} t / 2}+e^{-a_{k} t / 2}}{e^{a_{k} t / 2}-e^{-a_{k} t / 2}} \frac{d t}{t^{n}}\right),
$$


we have

$$
\begin{aligned}
& \sum_{k=0}^{n} \delta_{A W}\left(a_{k} ; a_{1}, \ldots, \check{a}_{k}, \ldots, a_{n} ;-1\right) \\
& =-1+\frac{1}{a_{0} \cdots a_{n}} \operatorname{res}_{t=0}\left(\prod_{k=0}^{n} a_{k} t \cdot \frac{e^{a_{k} t / 2}+e^{-a_{k} t / 2}}{e^{a_{k} t / 2}-e^{-a_{k} t / 2}} \frac{d t}{t^{n}}\right) \\
& \quad-8 \frac{1}{a_{0} \cdots a_{n}} \operatorname{res}_{t=0}\left(\prod_{k=0}^{n} \frac{a_{k} t}{e^{a_{k} t / 2}-e^{-a_{k} t / 2}} \cdot\left(\sum_{h=0}^{n}\left(e^{a_{h} t}+e^{-a_{h} t}\right)-2\right) \cdot \frac{d t}{t^{n}}\right) \\
& \quad+8\left(1+(-1)^{n}\right) \sum_{h ;-\alpha / 2+a_{h} \geq 0} \sharp\left\{\left(k_{0}, \ldots, k_{n}\right) \in \mathbb{Z}_{\geq 0}^{n+1} \mid \sum_{i=0}^{n} k_{i} a_{i}=-\alpha / 2+a_{h}\right\} \\
& \quad+32 \frac{1}{a_{0} \cdots a_{n}} \operatorname{res}_{t=0}\left(\prod_{k=0}^{n} \frac{a_{k} t}{e^{a_{k} t / 2}-e^{-a_{k} t / 2}} \frac{d t}{t^{n}}\right) .
\end{aligned}
$$

Note that for the last equality, the residue terms cancel by the cancellation formula applied to the complex 6-dimensional weighted projective space $\mathbb{C P}(\vec{a})$ of weight $\vec{a}=\left(a_{0}, a_{1}, \ldots, a_{6}\right)$, and we obtain the result (see Appendix A).

\section{Appendix A. The residue terms}

\section{A.1 The tangent $V$-bundle on $\mathbb{C P}(\vec{a})$}

The weighted projective space as a toric variety. In this section, we consider the weighted projective space $\mathbb{C P}(\vec{a})$ of weight $\vec{a}=\left(a_{0}, a_{1}, \ldots, a_{n}\right)$ defined by

$$
\mathbb{C P}(\vec{a})=\frac{\mathbb{C}^{n+1}-\{0\}}{\mathbb{C}^{*}},
$$

where $\mathbb{C}^{*}$ acts on $\mathbb{C}^{n+1}-\{0\}$ by

$$
t \cdot\left(z_{0}, \ldots, z_{n}\right)=\left(t^{a_{0}} z_{0}, \ldots, t^{a_{n}} z_{n}\right) .
$$

Then $\mathbb{C P}(\vec{a})$ admits a complex $n$-dimensional $V$-manifold structure with $n+1$ singular points which are cones over $(2 n-1)$-dimensional lens spaces.

In fact $\mathbb{C P}(\vec{a})$ can be described as a toric $V$-manifold as follows. Here, we employ the notation concerning toric varieties used by W. Fulton [1993]. Let $e_{1}, \ldots, e_{n}$ be linearly independent vectors, and let $e_{0}$ satisfy $e_{0}+e_{1}+\cdots+e_{n}=0$. Let $N$ be the lattice generated by $v_{0}, v_{1}, \ldots, v_{n}$ with $v_{j}=\left(1 / a_{j}\right) e_{j}$, let $M$ be the dual lattice $M=\operatorname{Hom}(N, \mathbb{Z})$, and denote the dual pairing by $\langle\rangle:, N \otimes_{\mathbb{Z}} M \rightarrow \mathbb{Z}$. For each set $J \subset I=\{0,1, \ldots, n\}$, let $\sigma_{J} \subset N_{\mathbb{R}}$ be the cone spanned by all the $v_{j}$ with $j \in J$ and define

$$
\sigma_{J}^{\vee}=\left\{m \in M \mid\langle m, v\rangle \geq 0, v \in \sigma_{J}\right\} .
$$


Then the set of cones $\Delta=\left\{\sigma_{J}\right\}_{J \subset I}$ define a fan whose corresponding toric variety $X(\Delta)$ is isomorphic to the weighted projective space $\mathbb{C P}(\vec{a})$. Each $n$-dimensional cone $\sigma_{I-\{k\}}$ corresponds to an open set $U_{\sigma_{I-\{k\}}}$ isomorphic to the quotient of $\mathbb{C}^{I-\{k\}}$ by the $\mathbb{Z} / a_{k}$ action on $\mathbb{C}^{I-\{k\}}$, that is,

$$
\zeta_{a_{k}}^{\ell} \cdot\left(z_{j}\right)_{j \neq k}=\left(\zeta_{a_{k}}^{a_{j} \ell} z_{j}\right)_{j \neq k}
$$

In fact, the coordinate ring of the neighborhood $U_{\sigma_{I-\{k\}}}$ is $\mathbb{C}\left[\sigma_{I-\{k\}}^{\vee} \cap M\right]$. If we take a sublattice $N_{k}=\sum_{j \neq k} v_{j} \subset N=N_{k}+\mathbb{Z} \cdot v_{k}$ and its dual $M_{k}=\operatorname{Hom}\left(N_{k}, \mathbb{Z}\right) \supset$ $M=\operatorname{Hom}(N, \mathbb{Z})$, we can, using the canonical duality pairing $N / N_{k} \times M_{k} / M \rightarrow$ $\mathbb{Q} / \mathbb{Z} \hookrightarrow \mathbb{C}^{*}$, define an action of $N / N_{k} \cong \mathbb{Z} / a_{k}$ on $\mathbb{C}\left[M_{k}\right]$ by

$\ell v_{k} \cdot \chi^{\sum_{j \neq k} m_{j} v_{j}^{*}}=\exp \left(\sum_{j \neq k} m_{j} v_{j}^{*}, \ell v_{k}\right) \chi^{\sum_{j \neq k} m_{j} v_{j}^{*}}=\exp \left(-\sum_{j \neq k} \frac{m_{j} a_{j} \ell}{a_{k}}\right) \chi^{\sum_{j \neq k} m_{j} v_{j}^{*}}$,

for $\ell v_{k} \in N$ and $\chi^{\sum_{j \neq k} m_{j} v_{j}^{*}} \in M_{k}$. Then the coordinate ring $\mathbb{C}\left[\sigma_{I-\{k\}}^{\vee} \cap M\right]$ is the $\mathbb{Z} / a_{k}$-invariant part of $\mathbb{C}\left[\sigma_{I-\{k\}}^{\vee} \cap M_{k}\right]=\mathbb{C}\left[\left\{\chi^{v_{j}^{*}}\right\}_{j \neq k}\right]$.

Divisors on the weighted projective space. Let $\tau=\sigma_{H}$ be the cone generated by $\left\{v_{j}\right\}_{j \in H}$ for some $H \subset I$, and define a lattice $N_{\tau}$ generated by $\tau \cap N$. Let $N(\tau)$ be the quotient lattice $N(\tau)=N / N_{\tau}$, and let $M(\tau)$ be its dual $M(\tau)=\tau^{\perp} \cap M$. Let $\bar{\sigma}_{J}$ be the quotient of $\sigma_{J}$ in $N(\tau)_{\mathbb{R}}$, that is,

$$
\bar{\sigma}_{J}=\left(\sigma_{J}+\left(N_{\tau}\right)_{\mathbb{R}}\right) /\left(N_{\tau}\right)_{\mathbb{R}} \subset N_{\mathbb{R}} /\left(N_{\tau}\right)_{\mathbb{R}}=N(\tau)_{\mathbb{R}} .
$$

Let $\operatorname{Star}(\tau)$ be the set of all $\bar{\sigma}_{J}$ such that the cones $\sigma_{J}$ have the cone $\tau=\sigma_{H}$ as a face, that is,

$$
\operatorname{Star}(\tau)=\left\{\bar{\sigma}_{J} \mid \sigma_{J} \succ \tau\right\}=\left\{\bar{\sigma}_{J} \mid H \subset J \subset I\right\} .
$$

Then the coordinate ring of $U_{\bar{\sigma}_{I-\{k\}}}$ is $\mathbb{C}\left[\sigma_{I-\{k\}}^{\vee} \cap M(\tau)\right]=\mathbb{C}\left[\sigma_{I-\{k\}}^{\vee} \cap \tau^{\perp} \cap M\right]$, and we have a surjection of rings $\mathbb{C}\left[\sigma_{I-\{k\}}^{\vee} \cap M\right] \rightarrow \mathbb{C}\left[\sigma_{I-\{k\}}^{\vee} \cap \tau^{\perp} \cap M\right]$, which corresponds to the fact that $U_{\bar{\sigma}_{I-\{k\}}}$ is isomorphic to the quotient of $\mathbb{C}^{I-\{k\} \cup H}$ by the $\mathbb{Z} / a_{k}$ action, that is,

$$
U_{\bar{\sigma}_{I-\{k\}}} \cong \frac{\mathbb{C}^{I-\{k\} \cup H}}{\mathbb{Z} / a_{k}} \hookrightarrow U_{\sigma_{I-\{k\}}} \cong \frac{\mathbb{C}^{I-\{k\}}}{\mathbb{Z} / a_{k}} .
$$

In fact, the corresponding toric variety $V(\tau)=X(\operatorname{Star}(\tau))$ with $\tau=\sigma_{H}$ is isomorphic to the weighted projective space $\mathbb{C P}\left(a_{I-H}\right) \subset \mathbb{C P}\left(a_{I}\right)$.

In particular, if $H=\{h\}$ and $\tau=\sigma_{\{h\}}$, then the corresponding toric variety $D_{v_{h}}=V(\tau)=X(\operatorname{Star}(\tau))$ is a divisor isomorphic to the weighted projective space $\mathbb{C P}\left(a_{I-\{h\}}\right) \subset \mathbb{C P}\left(a_{I}\right)$, and its normal $V$-bundle $N_{\mathbb{C P}\left(a_{I-\{h\}}\right) / \mathbb{C P}\left(a_{I}\right)}$ is isomorphic to 
the line $V$-bundle defined by

$$
\frac{\left(\mathbb{C}^{n+1}-\{0\}\right) \times \mathbb{C}}{\mathbb{C}^{*}} \rightarrow \frac{\mathbb{C}^{n+1}-\{0\}}{\mathbb{C}^{*}}
$$

with $\mathbb{C}^{*}$ action on $\left(\mathbb{C}^{n+1}-\{0\}\right) \times \mathbb{C}$ given by

$$
t \cdot\left(\left(z_{0}, \ldots, z_{n}\right), u\right)=\left(\left(t^{a_{0}} z_{0}, \ldots, t^{a_{n}} z_{n}\right), t^{a_{h}} \cdot u\right) .
$$

Now any Weil divisor can be written as $D_{\varphi}=\sum_{i \in I} \varphi\left(v_{i}\right) D_{v_{i}}$ for some $\varphi \in M=$ $\operatorname{Hom}(N, \mathbb{Z})$. Let $\psi \in M$ be a linear function defined by $\psi=a_{k} \sum_{i \in I-\{k\}} \varphi\left(v_{i}\right) v_{i}^{*}$, and define a divisor $\operatorname{div}\left(\chi^{\psi}\right)=\sum_{i \in I} \psi\left(v_{i}\right) D_{v_{i}}$. Then we can write

$$
a_{k} \cdot D_{\varphi}-\operatorname{div}\left(\chi^{\psi}\right)=\left(\sum_{i \in I} a_{i} \varphi\left(v_{i}\right)\right) D_{v_{k}} \in \mathbb{Z} \cdot D_{v_{k}},
$$

and we see that any divisor on $\mathbb{C P}(\vec{a})$ is linearly equivalent over the rationals to the rational divisor $\left(1 / a_{k}\right) D_{v_{k}}$, and if the $a_{i}$ are pairwise coprime integers then the group of all line $V$-bundles $\operatorname{Pic}_{V}(\mathbb{C P}(\vec{a}))$ is isomorphic to the infinite cyclic group generated by the line $V$-bundle associated with the divisor $\left(1 / a_{k}\right) D_{v_{k}}$, where $k$ is any index in $I=\{0, \ldots, n\}$. If we denote the associated line $V$-bundle by $H_{V}=\left[\left(1 / a_{k}\right) D_{v_{k}}\right]$ and call it the hyperplane line $V$-bundle, then we can write $\operatorname{Pic}_{V}(\mathbb{C P}(\vec{a}))=\mathbb{Z}\left[H_{V}\right]$. The divisor $D_{v_{\ell}}$ is linearly equivalent over the rationals to $\left(a_{\ell} / a_{k}\right) D_{v_{k}}$, and we can write $H_{V}=\left[\left(1 / a_{k}\right) D_{v_{k}}\right]=\left[\left(1 / a_{\ell}\right) D_{v_{\ell}}\right]$. In fact, $H_{V}$ is the line $V$-bundle on $\mathbb{C P}(\vec{a})$ defined by

$$
H_{V}=\frac{\left(\mathbb{C}^{n+1}-\{0\}\right) \times \mathbb{C}}{\mathbb{C}^{*}}
$$

with $\mathbb{C}^{*}$ action on $\left(\mathbb{C}^{n+1}-\{0\}\right) \times \mathbb{C}$ given by

$$
t \cdot\left(\left(z_{0}, \ldots, z_{n}\right), u\right)=\left(\left(t^{a_{0}} z_{0}, \ldots, t^{a_{n}} z_{n}\right), t \cdot u\right) .
$$

Intersections of divisors. Suppose $\sigma$ is a $k$-dimensional simplicial cone, and let $v_{1}, \ldots, v_{k}$ be the first lattice points along the edges of $\sigma$. Then the multiplicity mult $(\sigma)$ of $\sigma$ is defined to be the index of the lattice generated by the $v_{i}$ in the lattice $N_{\sigma}$ generated by $\sigma$, that is $\operatorname{mult}(\sigma)=\left[N_{\sigma}: \mathbb{Z} v_{1}+\cdots+\mathbb{Z} v_{k}\right]$. Let $V(\sigma)$ and $V(\tau)$ be the orbit closures of cones $\sigma$ and $\tau$, respectively, in a simplicial fan $\Delta$. Then the intersection $V(\sigma) \cdot V(\tau)$ can be calculated as follows (see [Fulton 1993, Section 5.1, p.100]):

$$
V(\sigma) \cdot V(\tau)=\left\{\begin{aligned}
\frac{\operatorname{mult}(\sigma) \cdot \operatorname{mult}(\tau)}{\operatorname{mult}(\gamma)} V(\gamma) & \text { if } \sigma \text { and } \tau \text { span the cone } \gamma, \text { with } \\
& \operatorname{dim} \sigma+\operatorname{dim} \tau=\operatorname{dim} \gamma ; \\
0 \quad & \text { if } \sigma \text { and } \tau \text { do not span a cone in } \Delta .
\end{aligned}\right.
$$


Note that any cone $\sigma_{J}$ is spanned by the linearly independent vectors $\left\{v_{j}\right\}_{j \in J}$ and hence the fan $\Delta=\left\{\sigma_{J} \mid J \subset I\right\}$ corresponding to $\mathbb{C P}(\vec{a})=X(\Delta)$ is simplicial. So we can apply the above formula to calculate intersection pairings. The multiplicity of $\sigma_{J}$ for any $J \subset I$ is

$$
\operatorname{mult}\left(\sigma_{J}\right)= \begin{cases}a_{i} & \text { if } J \cup\{i\}=I, \\ 1 & \text { otherwise }\end{cases}
$$

and the intersection $D_{v_{1}} \cdot D_{v_{2}} \cdots \cdots D_{v_{n-1}} \cdot D_{v_{n}}$ of divisors $D_{v_{i}}=V\left(\tau_{i}\right)$ for $\tau_{i}=\sigma_{\{i\}}$ can be calculated as

$$
\begin{aligned}
\left(D_{v_{1}} \cdot D_{v_{2}} \cdots D_{v_{n-1}}\right) \cdot D_{v_{n}} & =V\left(\sigma_{\{1, \cdots, n-1\}}\right) \cdot D_{v_{n}} \\
& =\frac{\operatorname{mult}\left(\sigma_{\{1, \cdots, n-1\}}\right) \cdot \operatorname{mult}\left(\sigma_{\{n\}}\right)}{\operatorname{mult}\left(\sigma_{\{1, \cdots, n\}}\right)} V\left(\sigma_{\{1, \cdots, n\}}\right) \\
& =\frac{1 \cdot 1}{a_{0}} V\left(\sigma_{\{1, \cdots, n\}}\right)=\frac{1}{a_{0}} V\left(\sigma_{I-\{0\}}\right) .
\end{aligned}
$$

The tangent $V$-bundle. Now we have the isomorphism

$$
T\left(\mathbb{C}^{n+1}-\{0\}\right) \cong\left(\mathbb{C}^{n+1}-\{0\}\right) \times \mathbb{C}^{n+1} .
$$

Hence for the left side we have $T\left(\mathbb{C}^{n+1}-\{0\}\right) / \mathbb{C}^{*} \cong T(\mathbb{C P}(\vec{a})) \oplus \mathbb{C}$. On the right, we have

$$
\frac{\left(\mathbb{C}^{n+1}-\{0\}\right) \times \mathbb{C}^{n+1}}{\mathbb{C}^{*}} \cong \bigoplus_{k=0}^{n} \frac{\left(\mathbb{C}^{n+1}-\{0\}\right) \times \mathbb{C}_{k}}{\mathbb{C}^{*}} \cong \bigoplus_{k=0}^{n} H_{V}^{a_{k}} .
$$

where $H_{V}$ is the hyperplane $V$-bundle on $\mathbb{C P}(\vec{a})$ defined above. Therefore we have the isomorphism $T(\mathbb{C P}(\vec{a})) \oplus \underline{\mathbb{C}} \cong \bigoplus_{k=0}^{n} H_{V}^{a_{k}}$.

Hence the canonical line $V$-bundle $K_{\mathbb{C P}(\vec{a})}$ is given by

$$
K_{\mathbb{C P}(\vec{a})}=\bigwedge^{n} T^{*}(\mathbb{C P}(\vec{a})) \cong \bigotimes_{k=0}^{n} H_{V}^{-a_{k}}=H_{V}^{-\sum_{k=0}^{n} a_{k}},
$$

and $\mathbb{C P}(\vec{a})$ admits an $V$-spin structure if and only if $\sum_{k=0}^{n} a_{k} \equiv 0(\bmod 2)$.

A.2 The characteristic numbers of $\mathbb{C P}(\overrightarrow{\boldsymbol{a}})$. Now we can evaluate the characteristic numbers as follows. First recall that $T(\mathbb{C P}(\vec{a})) \oplus \underline{\mathbb{C}} \cong \bigoplus_{k=0}^{n} H_{V}^{a_{k}}$, and the total Chern class is written as

$$
c(T(\mathbb{C P}(\vec{a})))=\prod_{k=0}^{n}\left(1+c_{1}\left(H_{V}^{a_{k}}\right)\right)=\prod_{k=0}^{n}\left(1+c_{1}\left(\left[D_{v_{k}}\right]\right)\right) .
$$


Then the evaluation of the $2 n$-dimensional cohomology class $c_{1}\left(H_{V}\right)^{n}$ on $\mathbb{C P}(\vec{a})$ can be calculated as

$$
\begin{aligned}
\int_{\mathbb{C P}(\vec{a})} c_{1}\left(H_{V}\right)^{n} & =\int_{\mathbb{C P}(\vec{a})} c_{1}\left(\left[\frac{D_{v_{0}}}{a_{0}}\right]\right)^{n}=\int_{\mathbb{C P}(\vec{a})} \bigwedge_{k=1}^{n} c_{1}\left(\left[\frac{D_{v_{k}}}{a_{k}}\right]\right) \\
& =\prod_{k=1}^{n} \frac{1}{a_{k}} D_{v_{k}}=\frac{1}{\prod_{k=1}^{n} a_{k}} \prod_{k=1}^{n} D_{v_{k}}=\frac{1}{\prod_{k=1}^{n} a_{k}} \cdot \frac{1}{a_{0}}=\frac{1}{\prod_{k=0}^{n} a_{k}} .
\end{aligned}
$$

Then it is easy to see that

$$
\begin{aligned}
\int_{\mathbb{C P}(\vec{a})} c_{1}\left(L^{a_{0}}\right)^{m_{0}} & \cdots c_{1}\left(L^{a_{n}}\right)^{m_{n}} c_{1}(L)^{m} \\
& =\frac{1}{\prod_{k=0}^{n} a_{k}} \operatorname{res}_{t=0}\left[\left(a_{0} t\right)^{m_{0}} \cdots\left(a_{n} t\right)^{m_{n}} \cdot t^{m}\right] \cdot \frac{d t}{t^{n+1}}=\frac{a_{0}^{m_{0}} \cdots a_{n}^{m_{n}}}{\prod_{k=0}^{n} a_{k}} .
\end{aligned}
$$

Hence the $\hat{A}$-genus of $\mathbb{C P}(\vec{a})$ is given by

$$
\begin{aligned}
\int_{\mathbb{C P}(\vec{a})} \hat{A}(T \mathbb{C P}(\vec{a})) & =\int_{\mathbb{C P}(\vec{a})} \prod_{k=0}^{n} \frac{c_{1}\left(H^{a_{k}}\right)}{e^{c_{1}\left(H^{a_{k}}\right) / 2}-e^{-c_{1}\left(H^{a_{k}}\right) / 2}} \\
& =\frac{1}{\prod_{k=0}^{n} a_{k}} \operatorname{res}_{t=0} \prod_{k=0}^{n} \frac{a_{k} t}{e^{a_{k} t / 2}-e^{-a_{k} t / 2}} \cdot \frac{d t}{t^{n+1}} .
\end{aligned}
$$

On the other hand, $L$-genus of $\mathbb{C P}(\vec{a})$ is given by

$$
\begin{aligned}
\int_{\mathbb{C P}(\vec{a})} L(T \mathbb{C P}(\vec{a})) & =\int_{\mathbb{C P}(\vec{a})} \prod_{k=0}^{n} \frac{e^{c_{1}\left(H^{a_{k}}\right) / 2}+e^{-c_{1}\left(H^{a_{k}}\right) / 2}}{e^{c_{1}\left(H^{a_{k}}\right) / 2}-e^{-c_{1}\left(H^{a_{k}}\right) / 2}} \\
& =\frac{1}{\prod_{k=0}^{n} a_{k}} \operatorname{res}_{t=0} \prod_{k=0}^{n} \frac{e^{a_{k} t / 2}+e^{-a_{k} t / 2}}{e^{a_{k} t / 2}-e^{-a_{k} t / 2}} \cdot \frac{d t}{t^{n+1}} .
\end{aligned}
$$

Next we want to evaluate $\int_{\mathbb{C P}(\vec{a})} \hat{A}(T \mathbb{C P}(\vec{a})) \operatorname{ch}\left(T^{\mathbb{C}}(\mathbb{C P}(\vec{a}))\right)$. First we note that

$$
\begin{aligned}
(T(\mathbb{C P}(\vec{a})) \oplus \underline{\mathbb{C}}) \otimes_{\mathbb{R}} \mathbb{C} & \cong T^{\mathbb{C}}(\mathbb{C P}(\vec{a})) \oplus \underline{\mathbb{C}} \otimes_{\mathbb{R}} \mathbb{C} \\
& \cong T^{\mathbb{C}}(\mathbb{C P}(\vec{a})) \oplus \underline{\mathbb{C}} \oplus \underline{\mathbb{C}} \\
& \cong \bigoplus_{k=0}^{n} H^{a_{k}} \otimes_{\mathbb{R}} \mathbb{C} \cong \bigoplus_{k=0}^{n} H^{a_{k}} \oplus H^{-a_{k}} .
\end{aligned}
$$

Now for the Chern character of the left side, we have

$$
\begin{aligned}
\operatorname{ch}(T(\mathbb{C P}(\vec{a})) \otimes \underline{\mathbb{C}}) & =\operatorname{ch}\left(T^{\mathbb{C}}(\mathbb{C P}(\vec{a})) \oplus \underline{\mathbb{C}} \oplus \underline{\mathbb{\mathbb { C }}}\right) \\
& =\operatorname{ch}\left(T^{\mathbb{C}}(\mathbb{C P}(\vec{a}))\right)+\operatorname{ch}(\underline{\mathbb{C}})+\operatorname{ch}(\underline{\mathbb{C}}) \\
& =\operatorname{ch}\left(T^{\mathbb{C}}(\mathbb{C P}(\vec{a}))\right)+2,
\end{aligned}
$$


and for the right, we have

$$
\operatorname{ch}(T(\mathbb{C P}(\vec{a})) \otimes \underline{\mathbb{C}})=\sum_{k=0}^{n} \operatorname{ch}\left(H^{a_{k}} \oplus H^{-a_{k}}\right)=\sum_{k=0}^{n}\left(e^{c_{1}\left(H^{a_{k}}\right)}+e^{-c_{1}\left(H^{a_{k}}\right)}\right) .
$$

Therefore we see that $\operatorname{ch}\left(T^{\mathbb{C}}(\mathbb{C P}(\vec{a}))\right)=\sum_{k=0}^{n}\left(e^{c_{1}\left(H^{a_{k}}\right)}+e^{-c_{1}\left(H^{a_{k}}\right)}\right)-2$. Thus we obtain the following equality.

$$
\begin{aligned}
\int_{\mathbb{C P}(\vec{a})} \hat{A}(T \mathbb{C P}(\vec{a})) \operatorname{ch}\left(T^{\mathbb{C}}(\mathbb{C P}(\vec{a}))\right) \\
=\int_{\mathbb{C P}(\vec{a})} \prod_{k=0}^{n} \frac{c_{1}\left(H^{a_{k}}\right)}{e^{c_{1}\left(H^{a_{k}}\right) / 2}-e^{-c_{1}\left(H^{a_{k}}\right) / 2}} \cdot\left(\sum_{k=0}^{n}\left(e^{c_{1}\left(H^{a_{k}}\right)}+e^{-c_{1}\left(H^{a_{k}}\right)}\right)-2\right) \\
=\frac{1}{\prod_{k=0}^{n} a_{k}} \operatorname{res}_{t=0} \prod_{k=0}^{n} \frac{a_{k} t}{e^{a_{k} t / 2}-e^{-a_{k} t / 2}} \cdot\left(\sum_{k=0}^{n}\left(e^{a_{k} t}+e^{-a_{k} t}\right)-2\right) \cdot \frac{d t}{t^{n+1}} .
\end{aligned}
$$

\section{References}

[Alvarez-Gaumé and Witten 1984] L. Alvarez-Gaumé and E. Witten, "Gravitational anomalies", Nuclear Phys. B 234:2 (1984), 269-330. MR 85j:81062

[Anderson et al. 1967] D. W. Anderson, E. H. Brown, Jr., and F. P. Peterson, "The structure of the Spin cobordism ring”, Ann. of Math. (2) 86 (1967), 271-298. MR 36 \#2160 Zbl 0156.21605

[Berndt 1978] B. C. Berndt, "Analytic Eisenstein series, theta-functions, and series relations in the spirit of Ramanujan”, J. Reine Angewandte Math. 303/304 (1978), 332-365. MR 80b:10035 Zbl 0384.10011

[Browder and Livesay 1967] W. Browder and G. R. Livesay, "Fixed point free involutions on homotopy spheres”, Bull. Amer. Math. Soc. 73 (1967), 242-245. MR 34 \#6781 Zbl 0156.21903

[Franz 1938] W. Franz, “Über die Torsion einer Überdeckung”, J. Reine Angew. Math. 173:245-254 (1938).

[Fukumoto 2002] Y. Fukumoto, "A generalized reciprocity law and w-invariants of lens spaces", Preprint, 2002, Available at http://isw3.kankyo-u.ac.jp/laboratory/fukumoto/research/preprints/ recipwinv20021128.pdf.

[Fukumoto 2005] Y. Fukumoto, "The index of the $\operatorname{Spin}^{c}$ Dirac operator on the weighted projective space and the reciprocity law of the Fourier-Dedekind sum", J. Math. Anal. Appl. 309:2 (2005), 674-685. MR 2006h:58026 Zbl 1079.58017

[Fukumoto and Furuta 2000] Y. Fukumoto and M. Furuta, "Homology 3-spheres bounding acyclic 4-manifolds", Math. Res. Lett. 7:5-6 (2000), 757-766. MR 2001m:57063 Zbl 0971.57026

[Fukumoto et al. 2001] Y. Fukumoto, M. Furuta, and M. Ue, “W-invariants and Neumann-Siebenmann invariants for Seifert homology 3-spheres”, Topology Appl. 116:3 (2001), 333-369. MR 2002j:57062 Zbl 0991.57019

[Fulton 1993] W. Fulton, Introduction to toric varieties, Annals of Mathematics Studies 131, Princeton University Press, NJ, 1993. The William H. Roever Lectures in Geometry. MR 94g:14028 Zbl 0813.14039

[Furuta 2001] M. Furuta, "Monopole equation and the 11/8-conjecture", Math. Res. Lett. 8:3 (2001), 279-291. MR 2003e:57042 Zbl 0984.57011 
[Han and Zhang 2004] F. Han and W. Zhang, "Modular invariance, characteristic numbers and $\eta$ invariants", J. Differential Geom. 67:2 (2004), 257-288. MR 2006k:58038 Zbl 05033771

[Hirzebruch and Zagier 1974] F. Hirzebruch and D. Zagier, The Atiyah-Singer theorem and elementary number theory, Mathematics Lectures 3, Publish or Perish, Boston, 1974. MR 58 \#31291 Zbl 0288.10001

[Kawasaki 1981] T. Kawasaki, "The index of elliptic operators over $V$-manifolds", Nagoya Math. J. 84 (1981), 135-157. MR 83i:58095 Zbl 0437.58020

[Lawson and Michelsohn 1989] H. B. Lawson, Jr. and M.-L. Michelsohn, Spin geometry, Princeton Mathematical Series 38, Princeton University Press, NJ, 1989. MR 91g:53001 Zbl 0688.57001

[Liu 1995] K. Liu, "Modular invariance and characteristic numbers", Comm. Math. Phys. 174:1 (1995), 29-42. MR 96m:57034 Zbl 0867.57021

[Milnor 1966] J. Milnor, "Whitehead torsion”, Bull. Amer. Math. Soc. 72 (1966), 358-426. MR 33 \#4922 Zbl 0147.23104

[Ochanine 1980/81] S. Ochanine, "Signature modulo 16, invariants de Kervaire généralisés et nombres caractéristiques dans la K-théorie réelle”, Mém. Soc. Math. France (N.S.) 5 (1980/81), 142. MR 83j:57014 Zbl 0462.57012

[Olum 1953] P. Olum, "Mappings of manifolds and the notion of degree", Ann. of Math. (2) 58 (1953), 458-480. MR 15,338a Zbl 0052.19901

[Reidemeister 1938] K. Reidemeister, "Homotopieringe und Linsenräume", Hamburger Abhandl. 11 (1938), 102-109.

[Rueff 1939] M. Rueff, "Beiträge zur Untersuchung der Abbildungen von Mannigfaltigkeiten", Compositio Math. 6 (1939), 161-202. MR 1557021

[Satake 1957] I. Satake, "The Gauss-Bonnet theorem for V-manifolds", J. Math. Soc. Japan 9 (1957), 464-492. MR 20 \#2022 Zbl 0080.37403

[Ue 2001] M. Ue, "On the intersection forms of Spin 4-manifolds bounded by spherical 3-manifolds", Algebr. Geom. Topol. 1 (2001), 549-578. MR 2003a:57048 Zbl 0992.57025

[Zagier 1972] D. B. Zagier, Equivariant Pontrjagin classes and applications to orbit spaces. Applications of the G-signature theorem to transformation groups, symmetric products and number theory, Lecture Notes in Mathematics 290, Springer, Berlin, 1972. MR 49 \#3965 Zbl 0238.57013

Received May 21, 2007. Revised September 22, 2007.

\section{YOSHIHIRO FUKUMOTO}

1-1-1 WAKABADAI-KITA

TOTTORI UNIVERSITY OF ENVIRONMENTAL STUdIES

TOTTORI

JAPAN

fukumoto@kankyo-u.ac.jp

http://isw3.kankyo-u.ac.jp/laboratory/fukumoto/index.html 